\title{
Spinal block at the cervico-medullary junction by a thickened transverse atlanto-axial ligament
}

\author{
Deb Kumar Mojumder MD PhD, Chuang-Kuo Wu MD PhD, \\ Ragesh Panikkath, MD, Nabeel S. Dar MD
}
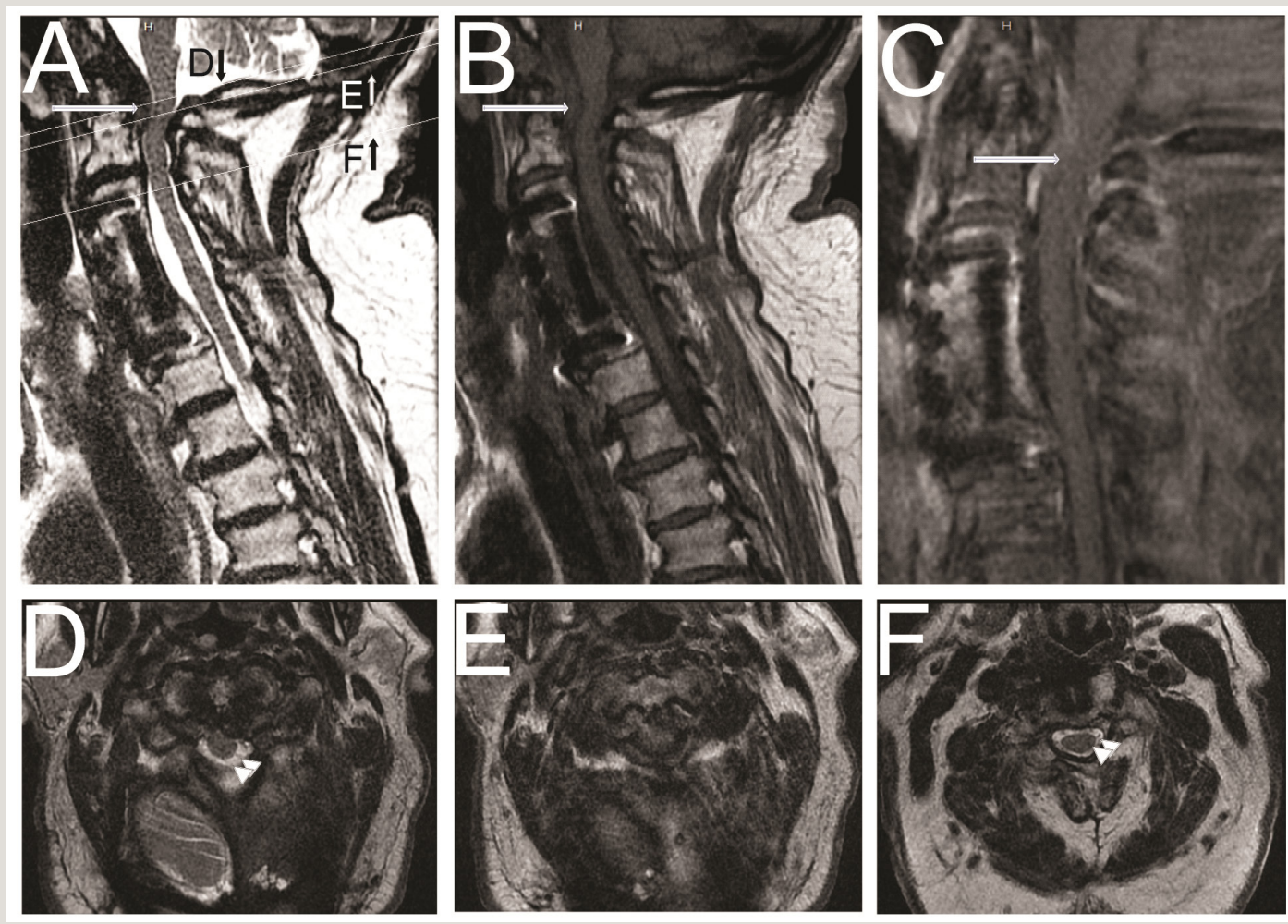

Figures Top panel: A: Sagittal T2 image of the cervical spine. Dotted lines marked D, E, and F indicate the horizontal sections shown in the bottom panel; B: Sagittal T1 image of the cervical spine; C: Sagittal T1 post-gadolinium image of the cervical spine. Arrows indicates the location of the thickened transverse atlanto-axial ligament causing deformity of the cervico-medullary junction anteriorly. This ligament is isointense to the neural tissue on T1-weighted sequence, is hypo-intense on T2-sequence, and is non-enhancing ${ }^{2}$. There are postsurgical changes of cervical spine reconstruction/ fusion from the $\mathrm{C} 3$ to the C6 level.

Bottom panel: $D, E$, and F represent horizontal sections indicated by the corresponding three dotted horizontal lines shown in A. The double arrowheads indicate the presence of T2 hyper-intense cerebrospinal fluid surrounding the cord that is missing at the region of the spinal block (E).

Corresponding author: Deb Kumar Mojumder MD Ph Contact Information: dkmj7@yahoo.com DOI: $10.12746 /$ swrccc 2014.0208.103

\section{CASE}

A 77-year-old man with underlying dementia was admitted with confusion, limited movement of his upper and lower extremities, low grade fever 
$\left(101.2^{\circ} \mathrm{F}\right)$, stiff neck, depressed deep tendon reflexes, and flexion plantar responses. Laboratory data were significant for leukocytosis, an elevated CK, and an elevated CRP. The patient was initially scheduled for a lumbar puncture procedure to evaluate him for meningitis pending imaging study results. His MRI of the cervical spine showed marked compression on the cervico-medullary junction by thickened transverse atlanto-axial ligament along with features suggesting complete spinal block (Figure 1). The patient was treated for meningitis without the lumbar puncture due to complete spinal block.

This case illustrates that a thickened transverse atlanto-axial ligament can present as quadriparesis. One should be cautious in performing lumbar puncture in cases of spinal block as this can aggravate signs of spinal cord disease. ${ }^{1}$ Surgical decompression via subtotal resection of the thickened transverse atlanto-axial ligament is the usual management of these cases. ${ }^{2}$ The patient's family deferred surgery. Over the next few days patient's confusion improved, but he continued to show limited movement in his extremities. He was subsequently discharged to a long term care facility. Two months after admission the patient showed improvement in both upper extremity and lower extremity weakness without surgery.

Author affiliation : Drs Mojumder and Wu are in the Department of Neurology at TTUHSC. Dr Panikkath is in the Department of Internal Medicine at TTUHSC. Dr Dar is a radiologist at University Medical Center, Lubbock, TX.

Submitted: $7 / 31 / 2014$

Accepted: 9/6/2014

Reviewers: Eman Attaya MD

Conflict of Interest: None

Published electronically: 10/15/2014

\section{References}

1. Habermann TM, editor. Mayo Clinic Internal Medicine Review 7ed: Page 703. CRC Press; 2006.

2. Ma JP, Ma L, You C, Liu JP. Cervicomedullary compression secondary to proliferation of transverse atlantal ligament. Neurol India 2012;60(1):125-6. 